enhanced, toll-like receptor 4-dependent T helper cell type 2 responses to inhaled antigen. J. Exp. Med. 196:1645-1651.

31. Braun-Fahrlander, C., et al. 2002. Environmental exposure to endotoxin and its relation to asthma in school-age children. N. Engl. J. Med. 347:869-877.

32. de Heer, H.J., Hammad, H., Kool, M., and Lambrecht, B.N. 2005. Dendritic cell subsets and immune regulation in the lung. Semin. Immunol. 17:295-303.

33. Brimnes, M.K., Bonifaz, L., Steinman, R.M., and Moran, T.M. 2003. Influenza virus-induced dendritic cell maturation is associated with the induction of strong $\mathrm{T}$ cell immunity to a coadministered, mormally nonimmunogenic protein. J. Exp. Med. 198:133-144.

34. Oriss, T.B., et al. 2005. Dynamics of dendritic cell phenotype and interactions with CD4+ T cells in airway inflammation and tolerance. J. Immunol. 174:854-863

35. Shortman, K., and Liu, Y.J. 2002. Mouse and human dendritic cell subtypes. Nat. Rev. Immunol. 2:151-161

36. Liu, Y.J. 2005. IPC: professional type 1 interferonproducing cells and plasmacytoid dendritic cell precursors. Annu. Rev. Immunol. 23:275-306.

37. Hawlisch, H., et al. 2005. C5a negatively regulates toll-like receptor 4 -induced immune responses. Immunity. 22:415-426.

38. Panina-Bordignon, P., et al. 2001. The C-C chemokine receptors CCR4 and CCR8 identify airway $\mathrm{T}$ cells of allergen-challenged atopic asthmatics. J. Clin. Invest. 107:1357-1364.

39. Hammad, H., et al. 2003. Monocyte-derived den- dritic cells exposed to Der p1 allergen enhance the recruitment of Th 2 cells: major involvement of the chemokines TARC/CCL17 and MDC/CCL22. Eur. Cytokine Netw. 14:219-228.

40. Kemper, C., et al. 2003. Activation of human CD4+ cells with CD3 and CD46 induces a T-regulatory cell 1 phenotype. Nature. 421:388-392.

41. Lewkowich, I.P., et al. 2005. CD4+CD25+ T cells protect against experimentally induced asthma and alter pulmonary dendritic cell phenotype and function. J. Exp. Med. 202:1549-1561.

42. Castellano, G., et al. 2004. Dendritic cells and complement: at the cross road of innate and adaptive immunity. Mol. Immunol. 41:133-140.

43. Gerard, N.P., et al. 2005. An anti-inflammatory function for the complement anaphylatoxin C5a-binding protein, C5L2. J. Biol. Chem. 280:39677-39680.

\title{
Good news in the nuclear envelope: loss of lamin A might be a gain
}

\author{
Paola Scaffidi and Tom Misteli
}

National Cancer Institute, National Institutes of Health, Bethesda, Maryland, USA.

\begin{abstract}
Genetic diseases often reveal the physiological roles of the affected proteins. The identification of mutations in the nuclear envelope proteins lamin A and lamin $\mathrm{C}$ as the cause of a diverse group of human diseases has expanded our understanding of the lamin proteins from being merely structural elements of the cell nucleus and has implicated them in novel cellular functions including signal transduction and gene expression. However, it now appears that the physiological relevance of one of the lamin proteins in organismal function has been overestimated. In this issue of the JCI, Fong et al. demonstrate that lamin A-deficient mice are phenotypically normal (see the related article beginning on page 743). The good news is these findings open the door to a new strategy for the therapeutic treatment of diseases caused by mutations in lamin A, such as muscular dystrophies and some types of premature aging syndromes.
\end{abstract}

Laminopathies are genetic diseases caused by mutations in the LMNA gene, which encodes lamins A and C (1). This diverse group of diseases includes several types of muscular dystrophies, lipodystrophies, and, curiously, premature aging diseases. One of the intriguing aspects of these diseases is that the affected gene encodes 2 of the most widely expressed structural proteins of the cell nucleus. Lamins A and $\mathrm{C}$ are intermediate filament-type proteins that, together with B-type lamins, form an extensive polymer network at the nuclear periphery (1). This nuclear lamina was long considered a merely passive

Nonstandard abbreviations used: FTI, farnesyltransferase inhibitor; HGPS, Hutchinson-Gilford progeria syndrome.

Conflict of interest: The authors have declared that no conflict of interest exists.

Citation for this article: J. Clin. Invest. 116:632-634 (2006). doi:10.1172/JCI27820. support structure for the cell nucleus but is now recognized as far more multifunctional and contributing to transduction of mechanical forces to the nucleus and to the nuclear periphery. In addition, the lamins are also present in the nuclear interior, where they have been implicated in organizing transcription, replication, and DNA repair (2).

No targeted therapies are available for laminopathies, and their molecular basis is poorly understood. One of the complications in analyzing lamin function is that lamins $\mathrm{A}$ and $\mathrm{C}$ are generated from the same gene, $L M N A$, by alternative splicing (3) (Figure 1A). Mature lamin A differs from lamin $\mathrm{C}$ by a 74-aa C-terminal addition and is generated from a precursor prelamin A protein. Prelamin A then undergoes extensive posttranslational processing, during which its $\mathrm{C}$ terminus to gene regulation via tethering of genes is modified by farnesylation, followed by endoproteolytic cleavage by the Zmpste24 protease (FACE1 in humans) (4). This farnesylation appears to be crucial since it promotes targeting of the lamin A protein to the nuclear periphery (5). Partially due to this elaborate processing mechanism, lamin A has long been considered the more important of the 2 isoforms, with lamin $\mathrm{C}$ merely playing a subordinate, auxiliary role. This view was recently reinforced by the discovery that mutations in LMNA that affect only lamin A, but not lamin $C$, led to accumulation of the farnesylated prelamin A intermediate, which acts in a dominant fashion to cause the premature aging disorder Hutchinson-Gilford progeria syndrome (HGPS) $(6,7)$. In this issue of the JCI, Fong et al. now directly challenge the notion of the paramount importance of lamin A by demonstrating that gene-targeted mice possessing only lamin C, but no lamin A or prelamin $\mathrm{A}$, are indistinguishable from wild-type mice (8).

\section{A lamin C-only mouse}

Fong et al. (8) generated "lamin C-only" mice (Lmna ${ }^{L C O / L C O}$ ) by creating a mutant Lmna allele that does not produce a prelamin A transcript. Based on growth rate, life span, bone structure, and muscle functionality, $L m n a^{L C O / L C O}$ mice appeared indistinguishable from wild-type mice, and histopathological analysis did not reveal any tissue abnormality. This is in 
A

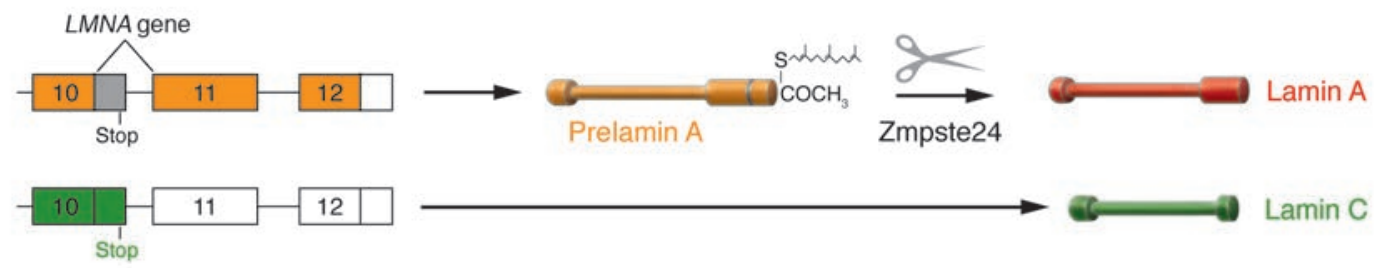

B

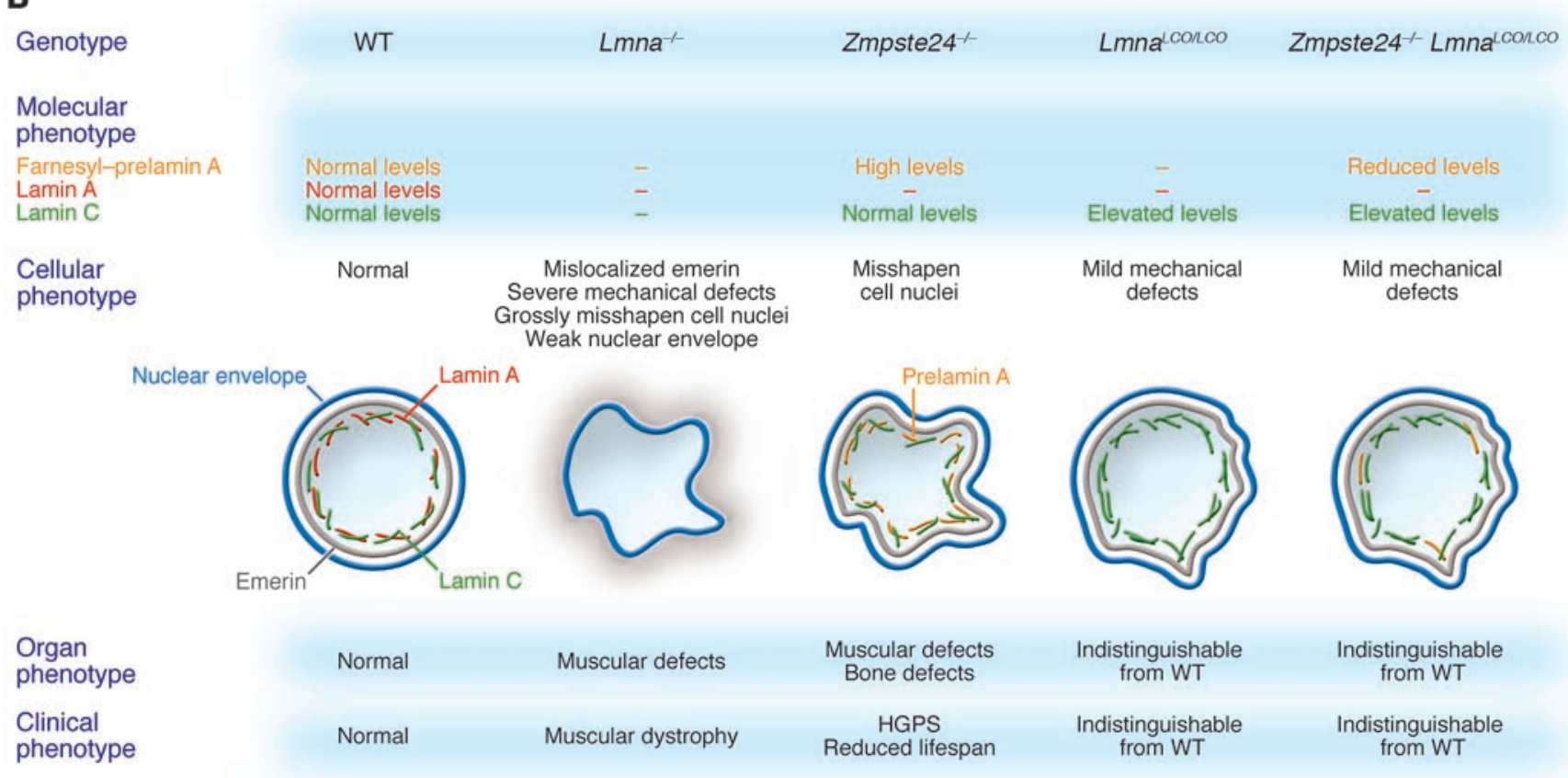

\section{Figure 1}

Lamin A is not essential for a healthy mouse. (A) Lamin A and lamin C are generated by alternative splicing of the LMNA gene. Use of a donor splice site within exon 10 generates the prelamin A protein, which is then modified by farnesylation and subsequently cleaved by the Zmpste24 endoprotease to produce mature lamin A. Skipping of the exon 10 splice site generates lamin $\mathrm{C}$. Due to the presence of a stop codon in exon 10, lamin C lacks the posttranslationally modified C-terminal region. (B) Comparison between cellular and organismal phenotypes in different mouse backgrounds.

striking contrast to $\mathrm{Lmna}^{-/-}$mice, which lack both lamin A and lamin $\mathrm{C}$ and show symptoms of muscular dystrophy and have a reduced life span (9) (Figure 1B). Clearly lamin $\mathrm{C}$ alone is sufficient to ensure a healthy mouse, and lamin $\mathrm{A}$ appears to have no essential function, at least under the experimental conditions used in this study. Regardless, an important corollary of the absence of an obvious phenotype in $\mathrm{Lmna}^{L C O / L C O}$ mice must be that the failure to generate mature lamin A, as observed in some laminopathies, is not the cause of these diseases, but rather that accumulation of lamin A intermediates or dominant-negative forms is the true problem. This point is elegantly reinforced by Fong et al. using Zmpste24-null mice, which lack the prelamin A endoprotease and fail to make mature lamin
A but accumulate a farnesylated prelamin A intermediate $(10,11)$. These mice display multiple premature aging symptoms reminiscent of HGPS. Remarkably, Zmpste24-/-Lmna $a^{L C O / L C O}$ mice were healthy despite the absence of mature lamin A (8). This effect is presumably due to the reduced levels of detrimental prelamin $\mathrm{A}$ in these mice (Figure 1B).

\section{The cellular life of lamin A}

The findings of Fong et al. (8) also shed light on the cellular behavior of lamin. First, they suggest that lamin $\mathrm{C}$ is able to functionally substitute for lamin $\mathrm{A}$, since nuclear morphological abnormalities typically seen in nuclei of cells from $\mathrm{Lmna}^{-/-}$ mice lacking both lamin A and lamin C were significantly reduced in $L m n a^{L C O / L C O}$ cells and practically absent in LmnaLCO/- cells (Figure 1B). Furthermore, the observed proper localization in $L m n a^{L C O / L C O}$ cells of lamin C itself as well as emerin, a nuclear envelope-associated protein that is mistargeted to the endoplasmic reticulum in $\mathrm{Lmna}^{-/-}$cells (9), clearly indicates that lamin A and lamin C are mostly redundant in the nucleus. However, a faint hint that maybe not all is perfectly well in $L m n a^{L C O} / L C O$ cells comes from the observation that although expression of lamin $\mathrm{C}$ resulted in significant correction of mechanical weaknesses characteristically seen in $\mathrm{Lmna}^{-/-}$cells (12), Lmna ${ }^{L C O / L C O}$ cells did not display full recovery of these properties, suggesting that lamin A might be important in the assembly of a robust lamin network.

These observations raise a particularly intriguing question: What is the relevance 
of the rather sophisticated sequence of prelamin A posttranslational modifications? It has generally been assumed that farnesylation of lamin A is important not only for its targeting to the nuclear periphery, but also for bringing lamin $\mathrm{C}$ to the lamina via interactions between lamin $\mathrm{A}$ and lamin $\mathrm{C}$ and for anchoring nuclear envelope-associated proteins such as emerin to the nuclear envelope $(13,14)$. However, in Lmna $a^{L C O} / L C O$ cells, lamin $C$ and emerin were able to properly localize to the nuclear envelope. One would suspect that the apparently superfluous and potentially harmful mechanism of posttranslational modifications of lamin A acts in a yet-to-be discovered aspect of its function. Obviously, since it appears that it is the accumulation of farnesylated prelamin A that is the true cause of some laminopathies, discovering the full function of the farnesyl group will be vital.

\section{A novel therapeutic approach for laminopathies}

The nonessential nature of lamin A opens up novel strategies for therapeutic intervention for lamin A-related diseases. Most laminopathies are heterozygous, and a major challenge in therapeutic approaches has been to specifically target the mutant lamin A without affecting the wild-type protein. Now that we know that loss of lamin A is not detrimental, its elimination, or elimination of lamin A precursors, from diseased cells seems like a safe therapeutic strategy. As proof of principle, Fong et al. (8) applied this idea to their Zmpste24 $4^{-/-}$mouse model of HGPS in which farnesyl-prelamin A accumulates. Using antisense oligonucleotides to knock down prelamin A, they demonstrate that reduction of prelamin A levels indeed had a positive effect and resulted in correction of morphological abnormalities of the cell nucleus in Zmpste $24^{-/-}$mouse embryonic fibroblasts. The next step will be to test whether systemic delivery of these oligonucleotides in mice will alleviate their premature aging symptoms. Similar results should also be achievable by RNA interference approaches. Elimination of lamin A might be a suitable therapeutic strategy not only for HGPS, but also for other laminopathies such as some forms of muscular dystrophy, although this approach might be limited to the group of laminopathies in which mutations only affect prelamin A or lamin A, but not lamin C.
The results reported in this issue by Fong et al. (8) are particularly encouraging for several emerging potential therapeutic approaches for HGPS. One such strategy is based on the oligonucleotide-mediated suppression of a cryptic pre-mRNA splice site in lamin A, whose activation leads to the production of the mutant prelamin A isoform in the first place (15). Since the cryptic splice site has high homology with the correct splice site within the same exon, there was a concern that the oligonucleotide would reduce global lamin A levels. A second promising strategy for the treatment of HGPS has been the use of farnesyltransferase inhibitors (FTIs) to block the farnesylation of prelamin A (4). Here the concern has been that this treatment might reduce levels of mature lamin A, since FTIs also inhibit the maturation of wild-type lamin A. In light of the dispensability of lamin A, these concerns are now greatly ameliorated for both approaches, although it is still unknown whether FTIs might have negative effects on other farnesylated proteins, such as the essential lamin B1 and lamin B2 proteins. While reduced levels of mutant prelamin A in HGPS patient cells has already been achieved by RNA interference (16), it will also be interesting to see whether knockdown of all lamin A leads to reversal of cellular defects in HGPS patient cells.

The results obtained with Lmna LCO/LCO mouse embryonic fibroblasts are encouraging with regard to their implication for therapy. Yet a word of caution is in order concerning possible limitations of this therapeutic approach in humans. Compelling evidence indicates that when it comes to lamin mutations, humans and mice behave differently. Many human laminopathies are caused by dominant heterozygous mutations. In contrast, in mouse models, comparable phenotypes are generally only observed in homozygous animals. For example, Lmna-/- mice develop muscular dystrophy, whereas their heterozygous counterparts are healthy (11). It thus remains to be seen how directly the behavior of lamins in mouse models can be applied to human laminopathies. Regardless, the important message remains: Lowering the amount of lamin A to reduce the production of toxic prelamin $\mathrm{A}$ is a reasonable compromise that might result in significant improvement of patient symptoms with no major side effects. The challenge will probably be to find the right balance to ensure a basal level of wild-type lamin A and at the same time reduce the toxic proteins below the critical threshold that leads to the diseases. In the case of some laminopathies, less lamin A might be more.

Address correspondence to: Tom Misteli, National Cancer Institute, National Institutes of Health, 41 Library Drive, Building 41, B610, Bethesda, Maryland 20892, USA. Phone: (301) 402-3959; Fax: (301) 496-4951; E-mail: mistelit@mail.nih.gov.

1. Broers, J.L., Hutchison, C.J., and Ramaekers, F.C. 2004. Laminopathies. J. Pathol. 204:478-488.

2. Shumaker, D.K., Kuczmarski, E.R., and Goldman, R.D. 2003. The nucleoskeleton: lamins and actin are major players in essential nuclear functions. Curr. Opin. Cell Biol. 15:358-366.

3. Lin, F., and Worman, H.J. 1993. Structural organization of the human gene encoding nuclear lamin A and nuclear lamin C. J. Biol. Chem. 268:16321-16326.

4. Young, S.G., Fong, L.G., and Michaelis, S. 2005. Thematic review series: lipid posttranslational modifications. Pre-lamin A, Zmpste24, misshapen cell nuclei, and progeria--new evidence suggesting that protein farnesylation could be important for disease pathogenesis. J. Lipid Res. 46:2531-2558.

5. Hennekes, H., and Nigg, E.A. 1994. The role of isoprenylation in membrane attachment of nuclear lamins. A single point mutation prevents proteolytic cleavage of the lamin A precursor and confers membrane binding properties. J. Cell Sci. 107:1019-1029.

6. De Sandre-Giovannoli, A., et al. 2003. Lamin a truncation in Hutchinson-Gilford progeria. Science. 300:2055.

7. Eriksson, M., et al. 2003. Recurrent de novo point mutations in lamin A cause Hutchinson-Gilford progeria syndrome. Nature. 423:293-298.

8. Fong, L.G., et al. 2006. Prelamin A and lamin A appear to be dispensable in the nuclear lamina. J. Clin. Invest. 116:743-752. doi:10.1172/JCI27125.

9. Sullivan, T., et al. 1999. Loss of A-type lamin expression compromises nuclear envelope integrity leading to muscular dystrophy. J. Cell Biol. 147:913-920.

10. Bergo, M.O., et al. 2002. Zmpste24 deficiency in mice causes spontaneous bone fractures, muscle weakness, and a pre-lamin A processing defect. Proc. Natl. Acad. Sci. U. S. A. 99:13049-13054.

11. Pendas, A.M., et al. 2002. Defective pre-lamin A processing and muscular and adipocyte alterations in Zmpste24 metalloproteinase-deficient mice. Nat. Genet. 31:94-99.

12. Lammerding, J., et al. 2004. Lamin A/C deficiency causes defective nuclear mechanics and mechanotransduction. J. Clin. Invest. 113:370-378. doi:10.1172/JCI200419670.

13. Raharjo, W.H., Enarson, P., Sullivan, T., Stewart, C.L., and Burke, B. 2001. Nuclear envelope defects associated with LMNA mutations cause dilated cardiomyopathy and Emery-Dreifuss muscular dystrophy. J. Cell Sci. 114:4447-4457.

14. Vaughan, A., et al. 2001. Both emerin and lamin C depend on lamin A for localization at the nuclear envelope. J. Cell Sci. 114:2577-2590.

15. Scaffidi, P., and Misteli, T. 2005. Reversal of the cellular phenotype in the premature aging disease Hutchinson-Gilford progeria syndrome. Nat. Med. 11:440-445.

16. Huang, S., et al. 2005. Correction of cellular phenotypes of Hutchinson-Gilford Progeria cells by RNA interference. Hum. Genet. 118:444-450. 